\title{
The effect of low cooling rates on dendrite morphology during directional solidification in Mg-Gd alloys: In situ X-ray radiographic observation
}

\author{
Yongbiao Wang ${ }^{\mathrm{a}, \mathrm{b}}$, Liming Peng a *, Yanzhou $\mathrm{Ji}^{\mathrm{b}}$, Xiaoxing Cheng ${ }^{\mathrm{b}}$, Nan Wang ${ }^{\mathrm{b}}$, Yan Zhao ${ }^{\mathrm{c}}$, Yanan \\ $\mathrm{Fu}^{\mathrm{d}}$, Long-Qing Chen ${ }^{\mathrm{b}}$ and Wenjiang Dinga
}

a National Engineering Research Center of Light Alloy Net Forming and State Key Laboratory of Metal Matrix Composite, Shanghai Jiao Tong University, Shanghai, 200240, China

b Department of Materials Science and Engineering, The Pennsylvania State University, University Park, PA 16802, USA

c School of Material Science and Engineering, Shanghai University, 200444 Shanghai, China

d Shanghai Synchrotron Radiation Facility, Shanghai Institute of Applied Physics, Shanghai, 215600, China

Corresponding author: Yongbiao Wang, E-mail : wsbiaoyongwang@163.com; Liming Peng, E-mail:

plm616@sjtu.edu.cn

The real time microstructure evolutions of directional solidification in $\mathrm{Mg}-\mathrm{Gd}$ alloys are obtained by synchrotron X-ray radiography, and the effects of different low cooling rates under a fixed thermal gradient are studied. Different from the organic alloys, the growth direction of columnar dendrites gradually rotates to the direction of the thermal gradient as the cooling rates increase, which is attributed to the difference of undercooling. Meanwhile, the interface velocity increases but the mean dendrite spacing decreases, and the morphology varies with implication for solute segregation.

Keywords: Mg-Gd alloys; Cooling rates; Directional solidification; X-ray radiography; Growth direction

\section{Introduction}

Nowadays, magnesium-rare earth alloys, which prominently enhance the yield strength and high-temperature creep resistance of the conventional Mg-alloys, have been widely applied in aerospace, automobile and aircraft industries [1-5]. In particular, Mg-Gd alloys have become an important category of strong lightweight structural casting alloys, as they exhibit better thermodynamic stability than other alloys [3]. However, compared with their counterparts of Al-alloys and steels, the mechanical properties of $\mathrm{Mg}$-Gd alloys still need further improvement. For this purpose, it is important to understand the effect of processing conditions on microstructure evolution, since the mechanical properties of Mg-Gd alloys can be manipulated through tailoring the microstructures. Notably, the casting conditions such as cooling rate, thermal gradient, and casting temperature play a key role in controlling the initial microstructures (i.e., the size, morphology and spacing of dendrites) in $\mathrm{Mg}-\mathrm{Gd}$ alloys. It is, therefore, critical to understand the effects of these different casting factors on the dendrites configurations.

Recently, Deschamps et al. [6] studied the dendrite morphology evolution under different solidification velocities in an organic alloy succinonitrile. Pang et al. [7] studied the microstructure and mechanical properties of Mg-Gd alloys under different cooling processes, such as quenching in warm water and cooling in different furnaces, but the cooling rates were too high to mimic the microstructure evolution feature during solidification. Paliwal et al. [8] investigated the effect of a large span of different cooling rates on the evolution of dendrite growth morphologies of $\mathrm{Mg}-\mathrm{Al}$ alloys with a focus on comparing the final microstructure such as grain size without analyzing the detailed mechanism of directional solidification under different low cooling rates. Furthermore, classical analysis, including quenching and decanting techniques, which is typically utilized in the above-mentioned studies, could not provide the real-time interface evolution and only gives a 'dead' picture of the solid microstructure, so that the important information about dendrite growth behaviors is usually lost. Although many computer simulation models have been proposed to model solidification microstructure [9], it is 
still challenging to predict the microstructure evolution under practical solidification conditions of a real alloy. The high-brilliance synchrotron X-radiation imaging technique based on phase-contrast has shown promise for in situ observations of evolving solidification microstructures [10-19]. To date, such experimental works on dendritic growth have been confined primarily to alloys with cubic structures, such as Al-Cu alloys [10-14], Sn alloys [15] and Fe-C alloys [16]. The solidification behavior of $\mathrm{Mg}-\mathrm{Gd}$ alloys has received considerably less attention. Recently, Wang et al $[18,19]$ used X-ray synchrotron tomographic technology to study the $\alpha-\mathrm{Mg}$ morphology evolution and dendritic orientation. However, only "frozen" pictures of dendrite microstructures during solidification were recorded, which still could not reflect dynamic evolution. In this paper, we present real time and visualized dendritic evolution during directional solidification of Mg- $6 \mathrm{wt} . \% \mathrm{Gd}$ alloys using in situ $\mathrm{X}$-ray synchrotron radiographic technique, which can not only help quantitatively analyze the dendritic morphology and interface velocity under different cooling rates, but also provide guidance for understanding the development of cast microstructures during solidification.

\section{Experiments}

The synchrotron X-ray radiography experiments were performed at beam line BL13W of SSRF (Shanghai, China). An X-ray energy of $20 \mathrm{keV}$ was employed, under which the mass X-ray absorption coefficients of $\mathrm{Mg}$ and $\mathrm{Gd}$ are 0.28 and $4.36 \mathrm{~m} 2 / \mathrm{kg}$ respectively [20], which can show a strong contrast between dendrites of $\alpha-\mathrm{Mg}$ and the $\beta$-Mg5Gd intermetallic obtained by radiographic technique. The nominal alloy composition was $\mathrm{Mg}-$ $6 \mathrm{wt} . \% \mathrm{Gd}$, and the size of the samples was $60 \mathrm{~mm}$ in length, $20 \mathrm{~mm}$ in width and $0.4 \mathrm{~mm}$ in thickness. The sample was sandwiched and sealed by high temperature adhesive between two 0.12-mm-thick $\mathrm{Al}_{2} \mathrm{O}_{3}$ plates. Solidification experiment was carried out inside a resistance furnace with two opposite windows. The temperature gradient along the crucible-sample length edge was measured by four embedded thermocouples 8 $\mathrm{mm}$ away from each other. An fixed temperature gradient $\mathrm{G}=5.0 \mathrm{~K} / \mathrm{mm}$ was calculated as the average value, and four different low cooling rates $0.033 \mathrm{~K} / \mathrm{s}, 0.1 \mathrm{~K} / \mathrm{s}, 0.25 \mathrm{~K} / \mathrm{s}$ and $0.5 \mathrm{~K} / \mathrm{s}$ was studied during the entire experiment. The sample was fixed upright in the holder of the furnace and heated to about $30 \mathrm{~K}$ higher than the melting point $915 \mathrm{~K}$ of the alloy and then cooled down. The X-ray energy penetrates the sample when solidification begins, and the information was collected by a CCD camera with typical explosion time $0.5 \mathrm{~s}$ per projection. The whole evolution process of dendrites during directional solidification was captured.

\section{Results and discussion}

Figure 1 shows the primary results of dendrite evolution under different low cooling rates. The white part represents $\alpha-\mathrm{Mg}$ dendrites. At the very beginning, the solid-liquid interface was mainly planar, and the initiation of instability began to develop due to small thermal perturbations. Some dendritic structures then evolved from the perturbed planar solidification front due to the different undercooling of the interfaces. Subsequently, the dendrites formed and competed with each other. The secondary dendrite and the tertiary arm grew with different levels due to the non-uniform temperature distributions. The color of the inter-dendritic region is obviously darker than dendrites from Figure 1, especially at later solidification times. It implies that the solute segregation occurred in the inter-dendritic region, and the solute concentration is higher than that in dendrites. Moreover, comparing the three groups of figures in Figure 1, with increasing cooling rate, the primary dendrites arm spacing obviously decreases, and the development of the secondary and tertiary dendrites arms is gradually restrained.

In order to qualitatively analyze the evolution of dendrites at different cooling rates, the interface moving velocity and the dependence of dendrite spacing on the system size were studied respectively. Figure 2(a) represents variation of the mean interface velocity. It shows that the interface moves faster as the cooling rates increase. Accordingly, the final steady-state spacing as a function of transverse sample size for four different cooling rates are shown in Figure 2(b). Since the growth rate of secondary arms can be negligible compared to that of the primary dendrite arm spacing (PDAS) during directional solidification, the curves just represent the spacing variation of the main trunks at different cooling rates. All the spacing curves increase at the beginning and finally reach a stable value, as shown in Figure 2(b). During this process, the dendrites can be blocked by 
the primary arm of an adjacent dendrite under the high cooling rate (Figure 1(c)) or by the secondary arm of the nearby dendrites under the low cooling rate (Figure 1(a)), and the final branch spacing is determined by the interplay between two different energy contributions[21]: one is the energy increase due to the growth of dendrites in different undesired directions, which plays a key role in blocking the variation of branch spacing; the other is the energy decrease due to the increase in the dendrite spacing, which provides the driving force for dendrites elimination. When these two effects balance, there will be no change for the branch spacing. Take the curve of $\mathrm{R}=0.033 \mathrm{~K} / \mathrm{s}$ as an example, the dendrite eliminating driving force plays a key role at the very beginning, which eventually leads to the PDAS increase. Then dendrites grow in different directions, which finally lead to the stable value of the PDAS.

Traditionally, a powder scaling of the form $\lambda=K G^{-a} V^{-b}$ [22] is usually assumed for theoretical analysis of steady-state PDAS in directional solidification, where $\lambda$ is the PDAS, $K$ is a constant of proportionality, $G$ and $V$ are temperature gradient and pulling rate respectively. In addition, the constant $K$ and exponents $a$ and $b$ typically vary in different theories. Kurz and Fisher [23] proposed a relation between the dendrite spacing $\lambda$ and the solidification velocity by using an elliptical approximation, but it could not describe the transient development of primary branches. Although other complex geometrical theories have also been suggested by considering growth regimes separately [24,25], their exponents are not unique over the entire regime of $G$ and $V$; moreover, their predictions about the geometric relation of directional solidification do not actually correspond to realistic casting situations. Experiments of solidified casts would suggest that PDAS should be covered using the notion of "ensemble averages", which captures their statistical nature [26]. In this work, the statistical data of PDAS are measured at different low cooling rates. Since the temperature gradient is fixed, the average PDAS follows a power-law relationship with respect to the cooling rate $R$, with the slope of the line in logarithmic scale to be close to -0.71, as shown in Figure 3, which shows a different result from former research work (about -0.5) [27]. But it indeed demonstrates that the PDAS obviously decreases when increasing the cooling rates.

In actual casting process, varying the cooling rates will lead to changes in primary dendrite arm spacing, growth direction and velocity spontaneously, since they are all interrelated. In this work, the growth direction of columnar dendrites rotates to the direction of the thermal gradient gradually as the cooling rates increase, as shown in Figure 1, which is different from the reported experimental results of succinonitrile alloy [6] and CBr4C2Cl6 alloy [28]. In [6] and [28], the cooling rates or the primary dendrites arm spacing are usually artificially fixed, and only one casting parameter is studied. The growth kinetics of dendrites is usually determined by the interplay between solute diffusion and the release of thermal energy. At the low cooling rate $0.033 \mathrm{~K} / \mathrm{s}$, solute diffusion plays a key role in the solidification process. The solid-liquid interface moved slowly and therefore the dendrite instability was triggered only for certain grain orientations with low surface stiffness. Hence, the growth direction of dendrites prefers the crystal direction $\langle 11 \overline{2} 0>$, as shown in Figure 1(a1). But as the cooling rate increases, the thermal release will dominate the solidification, and the undercooling of the interface becomes larger, which will directly lead to the changes of the dendritic growth direction and the interface velocity. Thus the original angle of the dendrites rotates to the direction of thermal gradient gradually, as shown in Figure 1(b1). When the cooling rate reaches $0.25 \mathrm{~K} / \mathrm{s}$, this trend is more obvious, as shown in Figure 1(c1).

\section{Conclusions}

In summary, we have studied the dendrite morphology evolution under different low cooling rates in directional solidification by using the synchrotron X-ray radiography. When the temperature gradient is fixed, the solidification velocity increases with increasing cooling rates, while the development of the secondary and tertiary dendrites arms is restrained. The dendrite growth direction gradually rotates to the direction of temperature gradient with the increase the cooling rate. The average primary dendrites arm spacing decreases with increasing cooling rate, which suggests that the relationship between the dendrite morphologies and the cooling rate is more complex than previously noted. This study provides a new sight into the dendritic morphology evolution and directional solidification in Mg-RE alloys. In addition, the experimental results can 
provide useful guidance for developing computational models to predict the microstructures and properties of casting alloys.

\section{Acknowledgments}

The work was supported by National Natural Science Foundation of China (No. 51304135, 51401118), New Century Excellent Talents in University of Ministry of Education of China (No. NCET-11-0329) and Program of Shanghai Subject Chief Engineering (No.14XD1425000). Y.B.W was financially supported by the Chinese Scholarship Council during his stay at PSU and Xiaoxing Cheng is supported by DOE through grant DE-FG0207ER46417.

\section{References}

[1] D. A. Edwards, J. Appl. Phys. 37, 561 (1966)

[2] W. Lefebvre, V. Kopp and C. Pareige, Appl. Phys. Lett. 100, 141906 (2012)

[3] Z. P. Lu, C. T. Liu, C. H. Kam and Y. Li, Appl. Phys. Lett. 82, 862 (2003)

[4] T. Honma, T. Ohkubo, S. Kamado, K. Hono, Acta Mater. 55, 4137 (2007).

[5] Q. Peng, Y. Huang, K.U. Kainer, Hort. Norbert, Mater. Lett. 83, 209 (2012).

[6] J. Deschamps, M. Georgelin, A. Pocheau, Phys. Rev. E. 78, 011605 (2008).

[7] S. Pang, et al. Mater. Sci. Eng. A. 562, 152 (2013).

[8] P. Manas, In-Ho Jung, Acta Mater. 13, 4848 (2013).

[9] M. Amoorezaei, S. Gurevich, N. Provatas, Acta Mater. 60, 657 (2012).

[10] N. Limodin, L. Salvo, M. Suery, M. DiMichiel, Acta Mater. 55, 3177 (2007).

[11] A. Bogno, H. Nguyen-Thi, A. Buffet, G. Reinhart, B. Billia, N. Mangelinck-Noe, N. Bergeon, J. Baruchel, T. Schenk, Acta Mater. 59, 4356 (2011).

[12] G. Reinhart, A. Buffet, H.Nguyen-Thi, B. Billia, H. Jung, N. Mangelinck-Noe, N. Bergeon, T. Schenk, J. Hartwig, J. Baruchel, Metall Mater Trans A. 39, 865 (2008).

[13] T.M. Wang, J.J. Xu, T.Q. Xiao, H.L. Xie, J. Li, T.J. Li, Z.Q. Cao, Phys Rev E. 81,042601 (2010).

[14] A.G. Murphy, D.J. Browne, W.U. Mirihanage, R.H. Mathiesen, Acta Mater. 61, 4559 (2013).

[15] H. Yasuda, I. Ohnaka, K. Kawasaki, A. Sugiyama, T. Ohmichi, J. Iwane, K. Umetani, J. Crystal Growth. 262,645 (2004).

[16] H. Yasuda, T. Nagira, M. Yoshiya, N. Nakatsuka, A. Sugiyama, K. Uesugi, K .Umetani, ISIJ. Int. 51, 402 (2011).

[17] A.G. Murphy, D.J. Browne, W.U. Mirihanage, R.H. Mathiesen, Acta Mater. 61, 4559 (2013).

[18] M.Y. Wang, J.J. Williams, L. Jiang, F. De Carlo, T. Jing and N. Chawlab, Scr. Mater. 65, 855 (2011).

[19] M.Y. Wang, Y.J. Xu, T. Jing, G.Y. Peng, Y.N. Fud and N. Chawla, Scr. Mater. 67, 629 (2012)

[20] NISTIR 5632, National Institute of Standards and Technology, Gaithersburg, MD (1995).

[21] M. Amoorezaei, S. Gurevich, N. Provatas, Acta Mater. 58, 6115 (2010). 
[22] J.D. Hunt. Solidification and casting of metals. London: The Metals Society (1979)

[23] W. Kurz, D.J. Fisher, Acta Mater. 29, 777 (1980).

[24] R. Trivedi and W. Kurz, Acta Mater. 42, 15 (1994).

[25] J.S. Kirkaldy, L.X. Liu, and A. Kroupa, Acta Mater. 43, 2905 (1995).

[26] N. Provatas, and K. Elder, John Wiley \& Sons, 2011

[27] M. Amoorezaei, S. Gurevich, N. Provatas, Acta Mater. 60, 657 (2012).

[28] S. Akamatsu, T. Ihle, Phys. Rev. E. 56, 4479 (1997).

\section{Figure Caption}

Fig.1 Microstructural evolution in solidification of Mg-6wt\%.Gd alloy under different cooling rates (a) $\mathrm{R}=0.033 \mathrm{~K} / \mathrm{s}$, , b) $\mathrm{R}=0.1 \mathrm{~K} / \mathrm{s}$ and (c) $\mathrm{R}=0.25 \mathrm{~K} / \mathrm{s}$ at different time, where t0 is the beginning time of solidification.

Fig.2 Dendritic morphology variation under four different cooling rates: (a) the average interface velocity changes, (b) the primary dendrites arm spacing changes at different transverse system sizes

Fig.3 The logarithm of statistical PDAR at the logarithm form of different cooling rates, when the temperature gradient is fixed at $5 \mathrm{~K} / \mathrm{mm}$. The slope of fitting line is -0.71 .

Fig.1
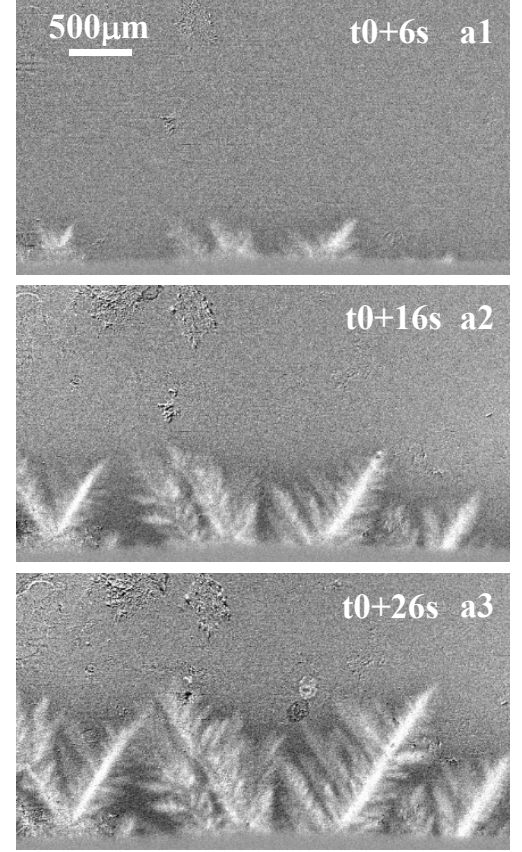

(a) $\mathrm{R}=0.033 \mathrm{~K} / \mathrm{s}$
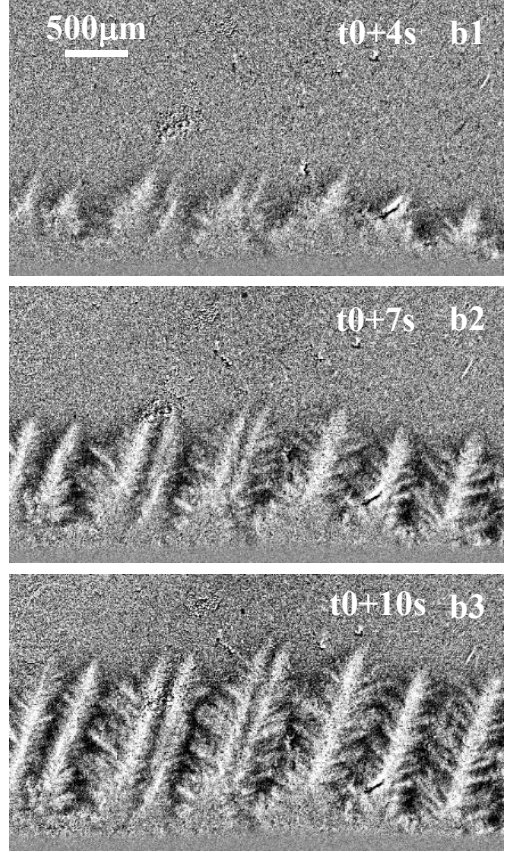

(b) $\mathrm{R}=0.1 \mathrm{~K} / \mathrm{s}$
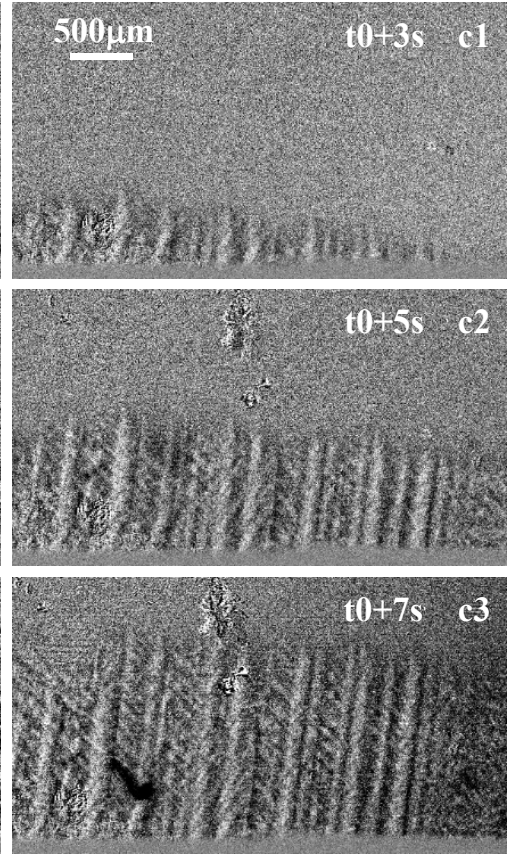

(c) $\mathrm{R}=0.25 \mathrm{~K} / \mathrm{s}$ 
Fig.2
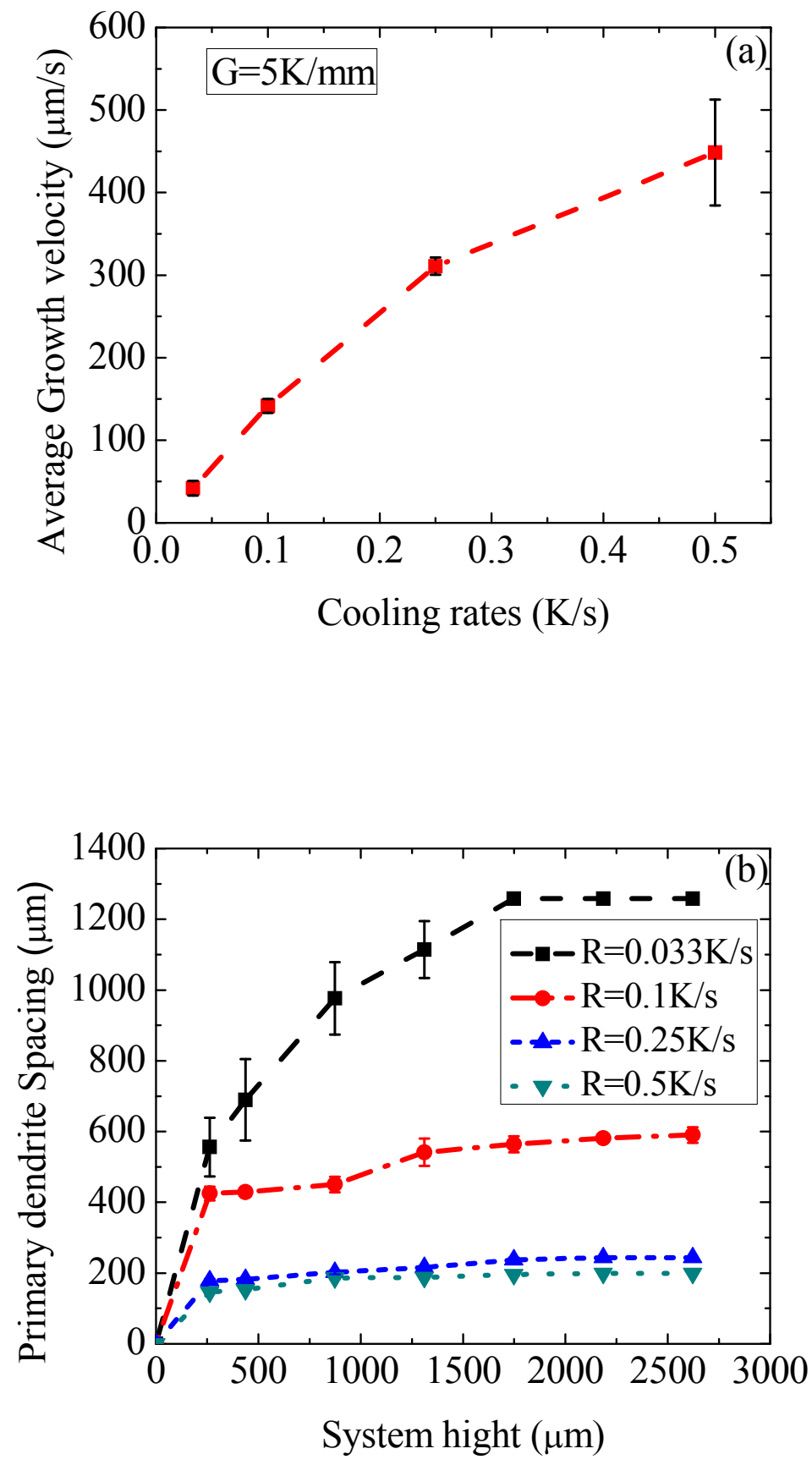
Fig.3

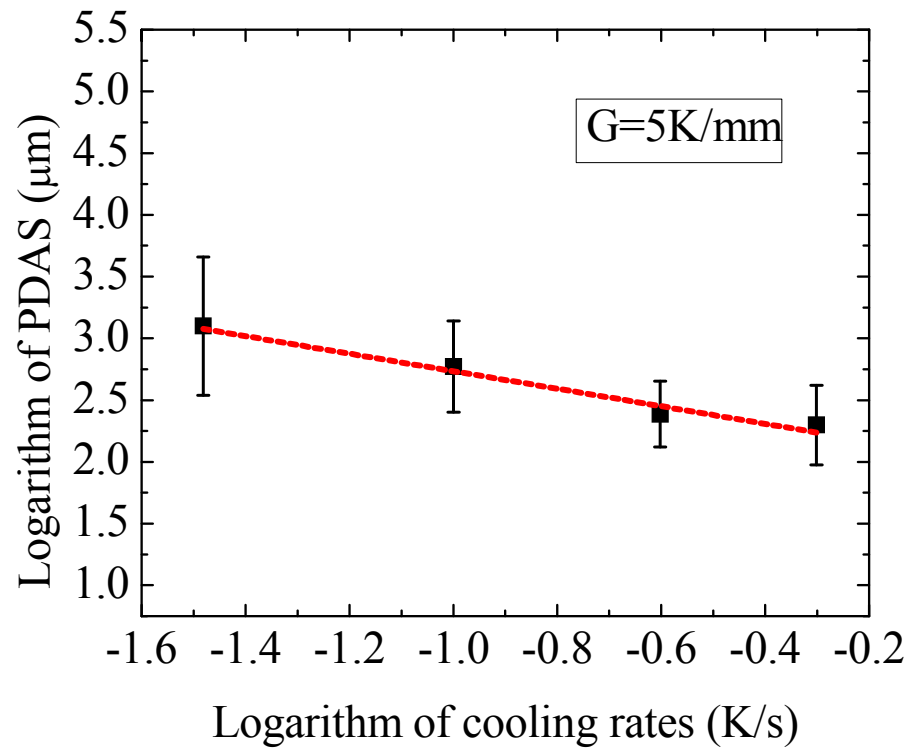

